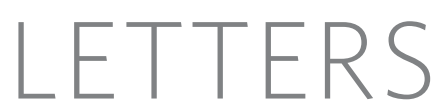

\title{
A dynamic upper atmosphere of Venus as revealed by VIRTIS on Venus Express
}

\author{
P. Drossart ${ }^{1}$, G. Piccioni ${ }^{2}$, J. C. Gérard ${ }^{4}$, M. A. Lopez-Valverde ${ }^{5}$, A. Sanchez-Lavega ${ }^{6}$, L. Zasova ${ }^{7}$, R. Hueso ${ }^{6}$, \\ F. W. Taylor ${ }^{8}$, B. Bézard ${ }^{1}$, A. Adriani ${ }^{3}$, F. Angrilli ${ }^{9}$, G. Arnold ${ }^{10}$, K. H. Baines ${ }^{11}$, G. Bellucci ${ }^{3}$, J. Benkhoff ${ }^{10}$, J. P. Bibring ${ }^{12}$, \\ A. Blanco ${ }^{13}$, M. I. Blecka ${ }^{14}$, R. W. Carlson ${ }^{11}$, A. Coradini ${ }^{3}$, A. Di Lellis ${ }^{15}$, T. Encrenaz ${ }^{1}$, S. Erard ${ }^{1}$, S. Fonti ${ }^{13}$, \\ V. Formisano ${ }^{3}$, T. Fouchet ${ }^{1}$, R. Garcia ${ }^{16}$, R. Haus ${ }^{10}$, J. Helbert ${ }^{10}$, N. I. Ignatiev ${ }^{7}$, P. Irwin $^{8}$, Y. Langevin ${ }^{12}$, S. Lebonnois ${ }^{17}$, \\ D. Luz ${ }^{1,19}$, L. Marinangeli ${ }^{18}$, V. Orofino ${ }^{13}$, A. V. Rodin ${ }^{7}$, M. C. Roos-Serote ${ }^{19}$, B. Saggin ${ }^{20}$, D. M. Stam ${ }^{21}$, D. Titov ${ }^{22}$, \\ G. Visconti ${ }^{23}$, M. Zambelli ${ }^{2}$, C. Tsang ${ }^{8} \&$ the VIRTIS-Venus Express Technical Team*
}

The upper atmosphere of a planet is a transition region in which energy is transferred between the deeper atmosphere and outer space. Molecular emissions from the upper atmosphere (90$120 \mathrm{~km}$ altitude) of Venus can be used to investigate the energetics and to trace the circulation of this hitherto little-studied region. Previous spacecraft ${ }^{1}$ and ground-based ${ }^{2-4}$ observations of infrared emission from $\mathrm{CO}_{2}, \mathrm{O}_{2}$ and $\mathrm{NO}$ have established that photochemical and dynamic activity controls the structure of the upper atmosphere of Venus. These data, however, have left unresolved the precise altitude of the emission ${ }^{1}$ owing to a lack of data and of an adequate observing geometry ${ }^{5,6}$. Here we report measurements of day-side $\mathrm{CO}_{2}$ non-local thermodynamic equilibrium emission at $4.3 \mu \mathrm{m}$, extending from 90 to $120 \mathrm{~km}$ altitude, and of night-side $\mathrm{O}_{2}$ emission extending from 95 to $100 \mathrm{~km}$. The $\mathrm{CO}_{2}$ emission peak occurs at $\sim 115 \mathrm{~km}$ and varies with solar zenith angle over a range of $\sim 10 \mathrm{~km}$. This confirms previous modelling ${ }^{7}$, and permits the beginning of a systematic study of the variability of the emission. The $\mathrm{O}_{2}$ peak emission happens at $96 \mathrm{~km} \pm 1 \mathrm{~km}$, which is consistent with three-body recombination of oxygen atoms transported from the day side by a global thermospheric sub-solar to anti-solar circulation, as previously predicted ${ }^{8}$.

Observations with the Visible and Infrared Thermal Imaging Spectrometer (VIRTIS) on Venus Express ${ }^{5,9}$ are obtained at wavelengths in the infrared between 1 and $5 \mu \mathrm{m}$ in the night side. These data cover the lower atmosphere up to the altitude of the upper cloud top at about $65 \mathrm{~km}$ and temperature profiles from the cloud tops to $\sim 90 \mathrm{~km}$ by the thermal inversion of the $\mathrm{CO}_{2} 4.3 \mu \mathrm{m}$ band. Also seen in the near-infrared range are night-side airglow emission from $\mathrm{O}_{2}$ (at $1.27 \mu \mathrm{m}$ ), and the non-local thermodynamic equilibrium (nonLTE) day-side emission by $\mathrm{CO}_{2}(4.3 \mu \mathrm{m})$ and $\mathrm{CO}(4.6 \mu \mathrm{m})$. This emission originates in the upper layers of the atmosphere between 90 and $140 \mathrm{~km}$, where pressures and collision rates are low, so that molecules in excited energy states can emit a photon before collisions bring them into thermal equilibrium. These observations were obtained both in limb geometry, where vertical variations are best investigated, and nadir geometry, where horizontal variations can be mapped in two dimensions. The highly eccentric $24 \mathrm{~h}$ orbit of Venus Express ${ }^{10}$ permits regular mapping of the southern hemisphere, on both the day and the night sides. The VIRTIS pixel size of $0.25 \mathrm{mrad}$ gives a spatial resolution of $15 \mathrm{~km}$ on Venus from apocentre, at a distance of about $66,000 \mathrm{~km}$.

The differences in $\mathrm{CO}_{2}$ day-side emissions in limb and nadir configurations are detailed in Fig. 1, showing the strong enhancement of the $4.3 \mu \mathrm{m}$ band at the limb, well above the cloud level, first observed by Galileo/Near Infrared Mapping Spectrometer ${ }^{6}$. This is a feature common to the three telluric planets with atmospheres, because Mars, Earth and Venus all exhibit non-LTE $\mathrm{CO}_{2}$ emission $^{11-13}$. The altitude of peak emission is defined by a competition between the exponentially increasing density of the atmosphere with depth (increasing the population of molecules that can emit) and the increasing collision frequency, which brings the molecules into LTE, quenching this emission. The altitude and the intensity of the peak emission are therefore related to the density structure of the atmosphere, and their observed variations to atmospheric behaviour, opening a new window on atmospheric processes at these altitudes.

A complete description of a non-LTE radiative transfer model that was originally developed for the Martian atmosphere ${ }^{14}$ but is relevant to these new observations of Venus can be found in recent updates ${ }^{6,7}$. The model simulates solar absorption and atmospheric thermal emission by $\mathrm{CO}_{2}$ molecules, including a large number of $\mathrm{CO}_{2}$ vibrational energy levels. It also includes a microphysical calculation of their state populations, with a detailed treatment of collisional processes. The probability that solar photons absorbed by a $\mathrm{CO}_{2}$ molecule and re-emitted resonantly will be reabsorbed at another level is included by using the Curtis matrix method. The standard profile of atmospheric temperature and pressure versus height is taken from the Venus International Reference Atmosphere (VIRA) ${ }^{15}$. As shown in Fig. 1, the model predicts the shape and peak altitude of the intensity quite well, and the fit is improved by suitable changes in the temperature-pressure profile, which affect not only the peak

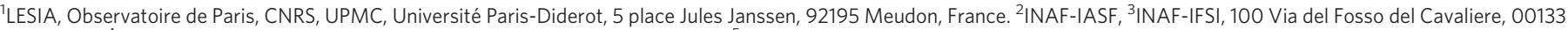

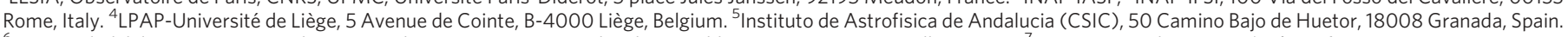

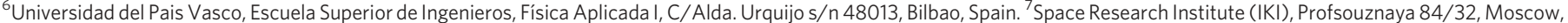

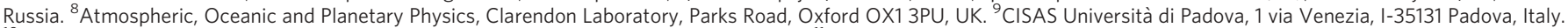

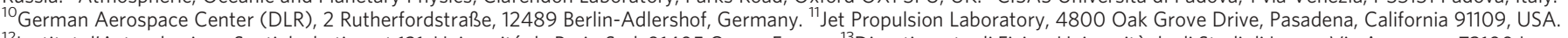

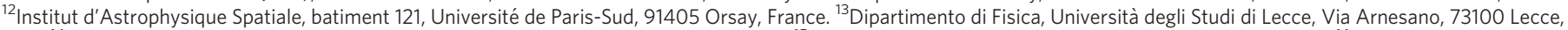

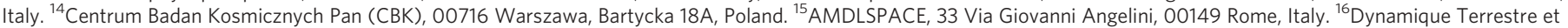

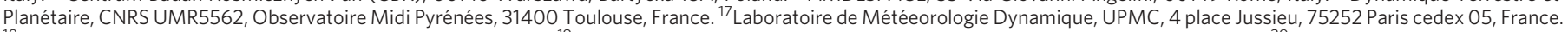

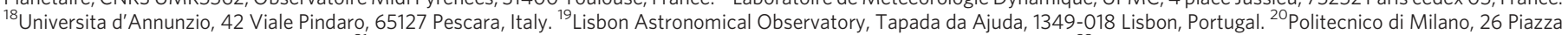

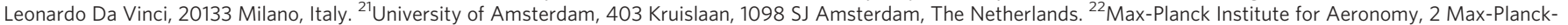
Strasse, 37191 Katlenburg-Lindau, Germany. ${ }^{23}$ University of I'Aquila, Dipartimento di Fisica, 10 Via Vetoio, 67010 Coppito I'Aquila, Italy.

*A list of participants and affiliations of the VIRTIS-Venus Express Technical Team appears at the end of the paper.
} 
altitude and intensity, but also the spectral shape of the observed emission. The fit of the model calculations to the observations is within a margin consistent with the estimated uncertainties in the model due to spectroscopy, input geophysical parameters and numerical approximations. Although a non-LTE retrieval from the observations is still beyond the scope of the present calculations, its future implementation will permit the atmospheric structure to be determined from limb observations.

In addition to the strong limb emissions, the planetary disk can be observed in nadir mode (Fig. 1a). With the radiative transfer model calibrated against the high-accuracy limb observations, future work will be devoted to the interpretation of the nadir emission. In particular, as the emission is sensitive to atmospheric density variations, it will be possible to search globally for density fluctuations at the altitude of peak emission, including those related to wave activity. Limb viewing geometry will still provide the best chance for the local detection of such waves, thanks to the strong enhancement of the emission due to the slant geometry.

Night-side $\mathrm{O}_{2}$ emissions at $1.27 \mu \mathrm{m}$ on Venus have been observed and mapped from the Earth ${ }^{8,16}$ since their first detection ${ }^{2}$. VIRTIS night-side observations complement these at much higher spatial resolution because the view from the Venus Express polar orbit covers the southern hemisphere from equator to pole. In addition, limb observations from an orbit around Venus give unprecedented access to the vertical distribution of the airglow layer, providing key constraints on the models. Figure 2 shows examples of the spectral and spatial distribution of $\mathrm{O}_{2}$ emission.
The $\mathrm{O}_{2}$ airglow in the near-infrared is produced by three-body recombination of oxygen atoms formed on the day side by photodissociation of $\mathrm{CO}_{2}$ and $\mathrm{CO}$. These oxygen atoms are transported to the night side by the global circulation, where they recombine according to the reaction:

$$
\mathrm{O}+\mathrm{O}+\mathrm{M} \rightarrow \mathrm{O}_{2}^{*}+\mathrm{M}
$$

where $\mathrm{O}_{2}$ * indicates one of the excited states of the $\mathrm{O}_{2}$ molecule and $\mathrm{M}$ is any neutral constituent. A fraction of the $\mathrm{O}_{2}$ molecules, estimated to be $\sim 7 \%$, is formed directly in the ${ }^{1} \Delta_{\mathrm{g}}$ metastable state. A substantial fraction of the upper states cascades into the ${ }^{1} \Delta_{\mathrm{g}}$ state, so that the net efficiency $\varepsilon$ of the production of this state in the threebody recombination may be considerably larger. We adopt here a value $^{8}$ of $\varepsilon=0.75$. Quenching of $\mathrm{O}_{2}{ }^{1} \Delta_{\mathrm{g}}$ molecules, mostly by collisions with $\mathrm{CO}_{2}$, can cause non-radiative transitions to the $\mathrm{O}_{2}$ ground state.

In Fig. 2c, the vertical distribution of the $\mathrm{O}_{2}\left({ }^{1} \Delta \mathrm{g}\right)$ emission rate calculated with a one-dimensional photochemical-diffusive transport model is compared with the limb profile observed on orbit 76 at $35^{\circ} \mathrm{N}$. The observations integrate all the $\mathrm{O}_{2}$ emission along the line of sight, which is accounted for in the model. A downward flux of oxygen atoms is allowed through the upper boundary, and the oxygen atom density decreases with depth below the peak according to reaction (1). The altitude of the peak of the $1.27 \mu \mathrm{m}$ emission is thus controlled by the competition between vertical transport and recombination, whereas the brightness is proportional to the downward
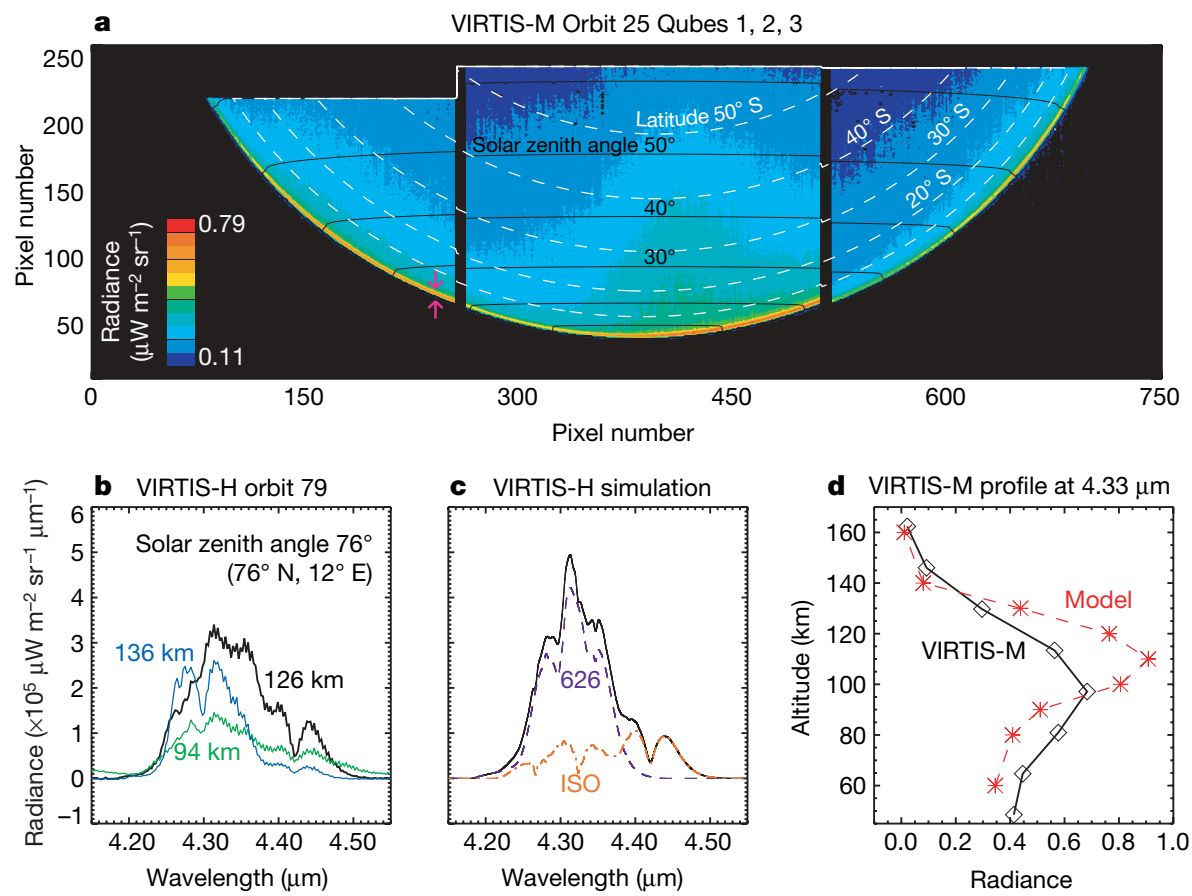

Figure $1 \mid \mathrm{CO}_{2}$ non-LTE emission on the day side of Venus. Example of $\mathrm{CO}_{2}$ observations around $4.3 \mu \mathrm{m}$ from VIRTIS. a, A VIRTIS-M (mapping channel) image of Venus at $4.33 \mu \mathrm{m}$ for Orbit 25 composed of three consecutive images (named Qubes 1-3), each with partial disk coverage, showing the $\mathrm{CO}_{2}$ emission enhanced from nadir to the limb. The variation of the peak emission along the limb is partly due to the solar zenith angle change, but additional variability is also present, which may be due to real atmospheric variability. Radiance (shown by colour scale) is integrated over the VIRTIS-M spectral resolution, which is $\sim 400$; the noise level is around 0.06 radiance units. The colour scale is linear between the extreme values, as indicated. b, Spectrum of VIRTIS-H (spectroscopic channel) during orbit 79 in a limb geometry at tangent heights and with a spectral resolution of $\sim 1,800$, with several emission peaks corresponding to different vibrational-rotational bands of $\mathrm{CO}_{2}$, whose relative weights depend on the saturation of each band, and therefore on the slant altitude of the observation. The noise radiance is $<1.5 \times 10^{4}$ units. c, Simulations of $\mathrm{CO}_{2}$ non-LTE emission at $126 \mathrm{~km}$ tangent height using a non-LTE radiative transfer model in standard atmospheric condition, adapted to the same illumination conditions and spectral resolution. Contributions from the main $\mathrm{CO}_{2}$ isotope (abbreviated 626) and from all other minor isotopes (abbreviated ISO) are indicated. Most of the features observed are captured by the model, but it seems to overestimate the peak radiance and to underestimate the relative contribution of the minor isotopes. $d$, An example of a limb emission profile, from the Venus disk at the location shown by the arrows in a. The vertical scale is the tangent altitude of the observation, and the highest emission corresponds to $\sim 100 \mathrm{~km}$. Simulation with the non-LTE radiative transfer model explains the limb brightening, but seems to overestimate the peak emission and its altitude. 
flux of oxygen atoms. The temperature and $\mathrm{CO}_{2}$ vertical distributions are again taken from the VIRA model ${ }^{15}$. Vertical transport is by molecular diffusion at high altitude and parameterized below the homopause by an eddy diffusion coefficient in the form $K=A / \sqrt{n} \mathrm{~cm}^{2} \mathrm{~s}^{-1}$, where $A$ is an empirically estimated parameter and $n$ is the total number density. The best fit to the profile corresponds to $A=2 \times 10^{12}$ and a downward flux of oxygen atoms of $F_{0}=5 \times 10^{11}$ atoms $\mathrm{cm}^{-2} \mathrm{~s}^{-1}$. The derived maximum oxygen atom number density, reached at $100 \mathrm{~km}$, is $1.3 \times 10^{11} \mathrm{~cm}^{-3}$. This quantity has not been measured previously by other techniques.

The derived value of $F_{0}$ can be compared with an average production of about $8 \times 10^{12}$ atoms $\mathrm{cm}^{-2} \mathrm{~s}^{-1}$ on the day side, such that if all atoms were carried to the night side without chemical loss, the average vertical emission rate would be 3 megaRayleigh (one megaRayleigh corresponds to the brightness of an extended source emitting $10^{12}$ photons $\mathrm{cm}^{-2} \mathrm{~s}^{-1}$ in $4 \pi$ steradians), that is, about seven times the value deduced from this limb profile. The geometry of the limb emission allows us to distinguish the flux from different layers, so the contribution of the reflection of the $\mathrm{O}_{2}$ emission by the cloud layer below can be neglected, unlike observations in nadir geometry where a factor of 1.75 enhancement has been derived $^{8}$. The non-homogeneous, time-dependent distribution of the $\mathrm{O}_{2}{ }^{1} \Delta_{\mathrm{g}}$ nightglow indicates that the local downward flow of oxygen may differ substantially from the mean value, in response to variations in factors such as the efficiency of the global day-tonight transport, the focusing effect of the night-side subsidence, changing zonal wind speeds, eddy transport efficiency, and gravity wave breaking.

Observations by VIRTIS in the nadir mode (Fig. 3) can be used to construct extensive maps of the Venus atmosphere in the $\mathrm{O}_{2}$ emission band. In nadir viewing, the contamination of the $\mathrm{O}_{2}$ emission by

the thermal emission of the deeper atmosphere has been subtracted to present pure $\mathrm{O}_{2}$ airglow images. The $\mathrm{O}_{2}$ night glow exhibits a large spatial and temporal variability, but the mean value, integrated over the night side of the southern hemisphere, is typically in the range of 0.8 megaRayleigh, which is in agreement with the early ground-based observations $^{2}$, which found 1.2 megaRayleigh for the night side. Recent ground-based observers have also seen variability as large as $20 \%$ over periods of hours ${ }^{16}$.

A new feature of these observations from Venus Express is the ability to observe these non-LTE and airglow phenomena from a vantage point above the South Pole, at an apocentre altitude of $66,000 \mathrm{~km}$. This unique perspective reveals the night-side emission over most of the southern hemisphere, from which the latitudinal distribution, and especially the distribution around local midnight, is obtained. Despite difficulties in interpretation of $\mathrm{O}_{2}$ variability in terms of bulk velocities, a search to derive the velocity fields from the large body of statistics in the full mission will be undertaken. The current observations are interpreted as showing signatures of the solar to antisolar circulation in the mesosphere. Although observations in the emission bands of $\mathrm{O}_{2}$ and $\mathrm{CO}_{2}$ sample similar altitude levels of the atmosphere, we emphasize that the differences in the processes involved give different insights into atmospheric models. The $\mathrm{CO}_{2}$ emission has a small dependence on atomic oxygen amount and is thought to be mostly regulated by a geographically simple solar illumination parameter, modulated by density variations like those expected from upward-propagating waves. A further dependence on the solar cycle is expected via the $\mathrm{O} / \mathrm{CO}_{2}$ ratio, and possibly via the thermal and density structure of the lower mesosphere. In contrast, the $\mathrm{O}_{2}$ emission is very sensitive to atomic oxygen amounts, and is driven by the highly variable and poorly understood dynamics of the global thermospheric sub-solar to anti-solar circulation
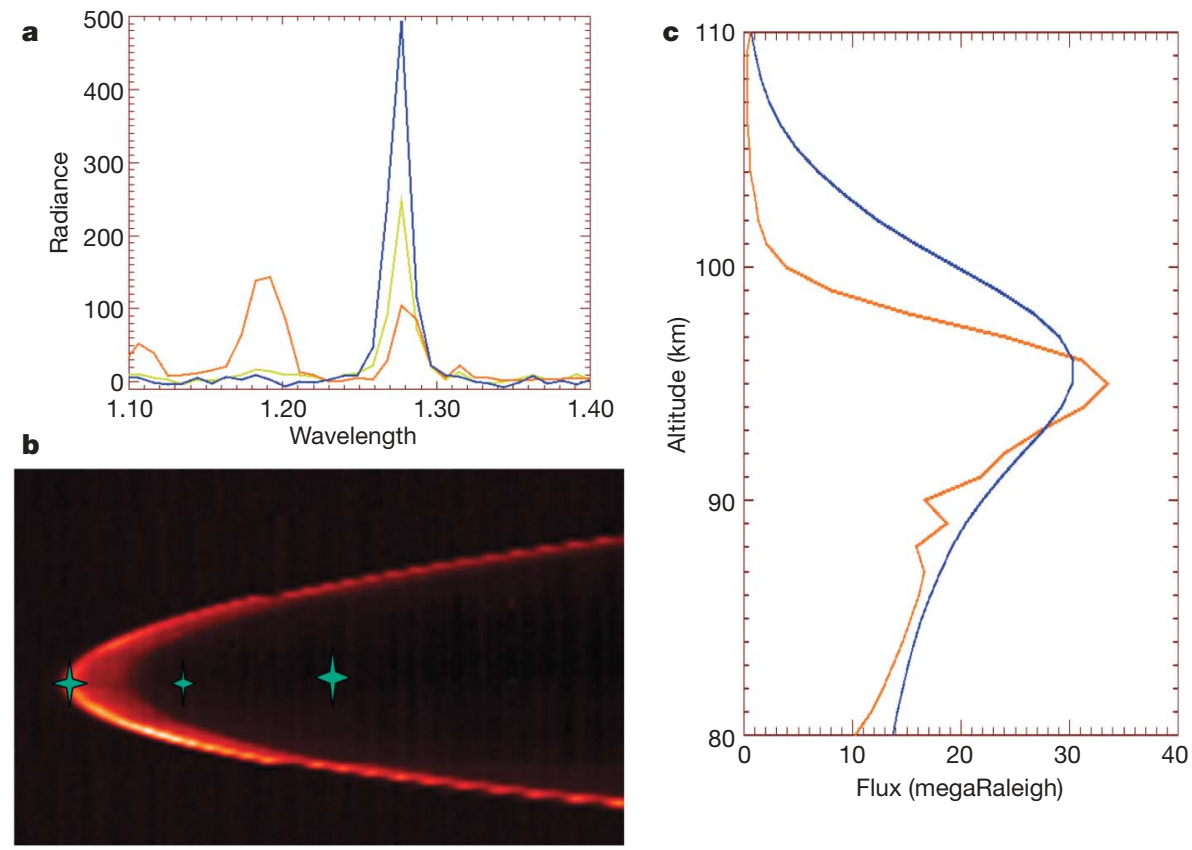

Figure $2 \mid \mathrm{O}_{2}$ emission in the night side upper atmosphere of Venus. a, Spectra of $\mathrm{O}_{2}$ emission at $1.27 \mu \mathrm{m}$; the three spectra show regions with dominant $\mathrm{O}_{2}$ emission (blue), intermediate $\mathrm{O}_{2}$ emission (green), and dominant thermal emission (red). The $\mathrm{O}_{2}$ emission is dominant at $1.27 \mu \mathrm{m}$, whereas thermal emission makes the major contribution in the adjacent spectral elements. Because the relative influence of the two contributions is very different in adjacent wavelength channels, the lower atmosphere contribution can be subtracted by linear interpolation to get the vertical profile of the $\mathrm{O}_{2}$ emission to first order. $\mathbf{b}$, Image of the limb at $1.27 \mu \mathrm{m}$ in orbit 76 by VIRTIS-M. In this reconstructed image, the horizontal dimension is the scan direction of VIRTIS-M. The limb was approached in the vertical dimension down to a minimum altitude of $\sim 80 \mathrm{~km}$, then the spacecraft motion was inverted, giving a second limb sounding of the atmosphere. The three stars give the path used for the retrieval of the vertical profiles of c. c, Limb vertical profile in $\mathrm{O}_{2}$ emission at a latitude of $35^{\circ} \mathrm{N}$, retrieved from the image, comparing the observed emission profile (red), with simulations from a one-dimensional model (blue). Peak altitude and intensities are well reproduced, but a smaller scale height is observed, compared to the model. 

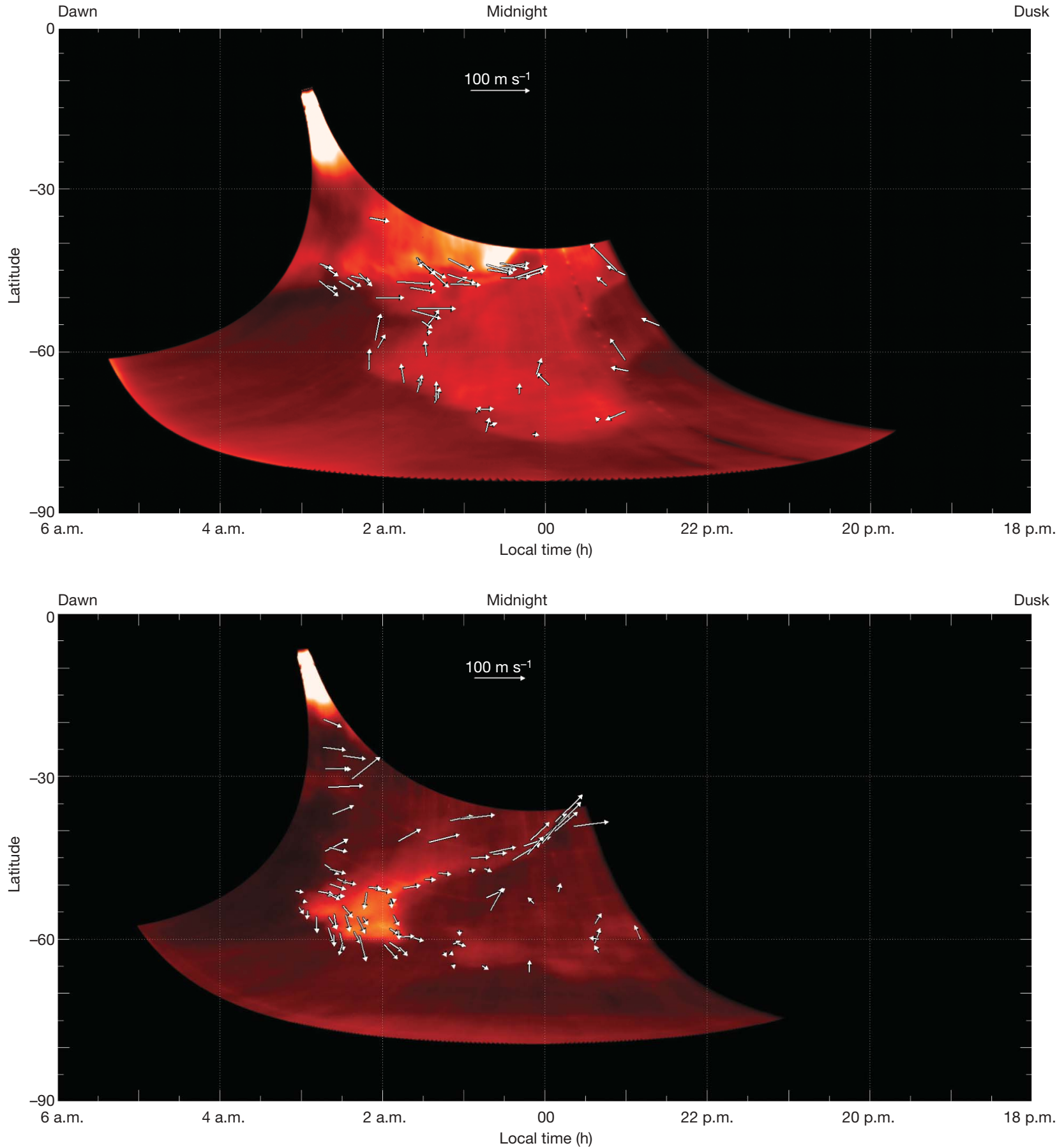

Figure $3 \mid$ Apparent motions in the $\mathbf{O}_{2}$ emission in nadir geometry during orbits 84 (above) and 96 (below). The images have been corrected for thermal emission from the deeper atmosphere, and projected in terms of the local time at each longitude. The maps exhibit almost pure $\mathrm{O}_{2}$ emission extending in the southern hemisphere from the equatorial zone to high latitudes. For each orbit a sequence of two images separated by $\sim 1 \mathrm{~h}$ shows the displacement of the emitted structures. The airglow apparent motions have velocities of the order of $60 \mathrm{~m} \mathrm{~s}^{-1}$. The flow is dominated by meridional motions from polar latitudes equatorward and by a zonal flow from dawn to midnight. Both are signatures of the solar to antisolar circulation of the mesosphere. Repetition of such images from orbit to orbit shows a high degree of variability and only a statistically large set of observations will provide a global view of the general characteristics of the $\mathrm{O}_{2}$ emission over the full Venus Express mission.
(Fig. 3). The systematic observations now underway should reveal the detailed characteristics of the latter and its dependence on solar activity.

\section{Received 9 April; accepted 27 July 2007.}

1. Bougher, S. W., Alexander, M. J. \& Mayr, H. G. Upper atmosphere dynamics: global circulation and gravity waves. In Venus II: Geology, Geophysics, Atmosphere, and Solar Wind Environment (eds Bougher, S. W. Hunten, D. M. \& Philips R. J.) 259-292 (Univ. Arizona Press, Tucson, 1997).
2. Connes, P. Noxon, J. F., Traub, W. A. \& Carleton, N. $\mathrm{O}_{2}{ }^{1} \Delta$ emission in the day and night airglow of Venus. Astrophys. J. Lett. 233, L29-L32 (1979).

3. Crovisier, J. et al. Carbon monoxide emissions at $4.7 \mu \mathrm{m}$ from Venus' atmosphere. Planet. Space Sci. 54, 1398-1414 (2006).

4. Lellouch, E. et al. Monitoring of mesospheric structure and dynamics. In Venus II: Geology, Geophysics, Atmosphere, and Solar Wind Environment (eds Bougher, S. W. Hunten, D. M. \& Philips R. J.) 295-324 (Univ. Arizona Press, Tucson, 1997).

5. Drossart, P. et al. Scientific goals for the observation of Venus by VIRTIS on ESA/Venus Express mission. Planet. Space Sci. doi:10.1016/j.pss.2007.01.03 (in the press). 
6. Lopez-Valverde, M. A., Drossart, P., Carlson, R., Mehlman, R. \& Roos-Serote, M Non-LTE infrared observations at Venus: From NIMS/Galileo to VIRTIS/Venus Express. Planet. Space Sci. doi:10.1016/j.pss.2007.01.008 (in the press) (2007)

7. Roldan, C., Lopez-Valverde, M. A., Lopez-Puertas, M. \& Edwards, D. P. Non-LTE infrared emissions of $\mathrm{CO}_{2}$ in the atmosphere of Venus. Icarus 147, 11-25 (2000).

8. Crisp, D. et al. Ground-based near-infrared observations of the Venus nightside: 1.27- $\mu \mathrm{m} \mathrm{O}_{2}\left({ }^{1} \Delta_{\mathrm{g}}\right)$ airglow from the upper atmosphere. J. Geophys. Res. 101 4577-4594 (1996)

9. Piccioni, G. et al. South-polar features on Venus similar to those near the north pole. Nature doi:10.1038/nature06209 (this issue).

10. Titov, D. V. et al. Venus Express science planning. Planet. Space Sci. 54, 1279-1297 (2006).

11. Lellouch, E. et al. The $2.4-45 \mu \mathrm{m}$ spectrum of Mars observed with the infrared space observatory. Planet. Space Sci. 48, 1393-1405 (2000).

12. Picard, R. H., Wintersteiner, P. P., Hegblom, E. R. \& Richards, E. Remote sensing of discrete stratospheric gravity-wave structure at $4.3-\mu \mathrm{m}$ from the MSX satellite. Geophys. Res. Lett. 25, 2809-2812 (1998).

13. Formisano, V., Maturilli, A., Giuranna, M., D'Aversa, E. \& Lopez-Valverde, M. A. Observations of non-LTE emission at 4-5 microns with the planetary Fourier spectrometer aboard the Mars Express mission. Icarus 182, 51-67 (2006).

14. Lopez-Valverde, M. A. \& Lopez-Puertas, M. A non-local thermodynamic equilibrium radiative transfer model for infrared emissions in the atmosphere of Mars. 1. Theoretical basis and nighttime populations of vibrational states. J. Geophys. Res. 99, 13093-13115 (1994).

15. Keating, G. M. et al. Models of Venus neutral upper atmosphere: Structure and composition. Adv. Space Res. 5, 117-171 (1985).

16. Ohtsuki, S. et al. Ground-based observation of the Venus 1.27- $\mathrm{m} \mathrm{O}_{2}$ airglow. Adv Space Res. 36, 2038-2042 (2005).

Acknowledgements We acknowledge the work of the entire Venus Express team of ESA and Astrium, who allowed these data to be obtained. This work is supported by the national space agencies CNES and ASI, and by SFTC in the UK. J.C.G. thanks the FNRS and the PRODEX-ESA program for funding.
Author Contributions P.D. and G.P. have coordinated the work as Principal Investigators of VIRTIS. J.C.G. contributed to the $\mathrm{O}_{2}$ model, L.Z. to the $\mathrm{O}_{2}$ dat selection, and M.A.L.-V. to the $\mathrm{CO}_{2}$ model. A.S.-L. and R.H. contributed to the dynamics calculation for Fig. 3. All authors contributed equally to the planning of the work, instrumental calibration and data calibration. F.W.T. and B.B. helped to finalize the paper.

Author Information Reprints and permissions information is available at www.nature.com/reprints. The authors declare no competing financial interests. Correspondence and requests for materials should be addressed to P.D. (pierre.drossart@obspm.fr).

*The VIRTIS-Venus Express Technical Team Eleonora Ammannito ${ }^{24}$, Alessandra Barbis $^{25}$, Rainer Berlin ${ }^{26}$, Carlo Bettanini ${ }^{27}$, Angelo Boccaccini ${ }^{24}$, Guillaume Bonnello ${ }^{29}$, Marc Bouyé ${ }^{30}$, Fabrizio Capaccioni ${ }^{31}$, Alejandro Cardesin ${ }^{31}$, Francesco Carraro $^{32}$, Giovanni Cherubini ${ }^{25}$, Massimo Cosi ${ }^{25}$, Michele Dami ${ }^{25}$, Maurizio De Nino $^{32}$, Davide Del Vento ${ }^{24}$, Marco Di Giampietro ${ }^{25}$, Alessandro Donati ${ }^{25}$, Olivier Dupuis $^{30}$, Sylvie Espinasse ${ }^{31}$, Anna Fabbri ${ }^{25}$, Agnès Fave ${ }^{30}$, lacopo Ficai Veltroni ${ }^{25}$, Gianrico Filacchione ${ }^{31}$, Katia Garceran ${ }^{30}$, Yamina Ghomchi ${ }^{30}$, Maurizio Giustizi ${ }^{25}$, Brigitte Gondet ${ }^{29}$, Yann Hello ${ }^{30}$, Florence Henry ${ }^{30}$, Stefan Hofer ${ }^{33}$, Gerard Huntzinger ${ }^{30}$, Juergen Kachlicki ${ }^{26}$, René Knoll ${ }^{30}$, Driss Kouach ${ }^{30}$, Alessandro Mazzoni ${ }^{25}$, Riccardo Melchiorri ${ }^{30}$, Giuseppe Mondello ${ }^{25}$, Francesco Monti ${ }^{32}$, Christian Neumann ${ }^{33}$, Fabrizio Nuccilli ${ }^{28}$, Jérôme Parisot ${ }^{30}$, Claudio Pasqui $^{25}$, Stefano Perferi ${ }^{25}$, Gisbert Peter ${ }^{26}$, Alain Piacentino ${ }^{30}$, Carlo Pompei ${ }^{25}$, Jean-Michel Réess ${ }^{30}$, Jean-Pierre Rivet $^{30}$, Antonio Romano ${ }^{25}$, Natalie Russ ${ }^{26}$, Massimo Santoni ${ }^{25}$, Adelmo Scarpelli ${ }^{25}$, Alain Sémery ${ }^{30}$, Alain Soufflot ${ }^{29}$, Douchane Stefanovitch ${ }^{30}$, Enrico Suetta ${ }^{25}$, Fabio Tarchi $^{25}$, Nazzareno Tonetti ${ }^{25}$, Federico Tosi ${ }^{28}$ \& Bernd Ulmer ${ }^{26}$

Affiliations for participants: ${ }^{24}$ INAF-IFSI, 00133 Rome, Italy. ${ }^{25}$ Galileo Avionica, Florence, 50013, Italy. ${ }^{26}$ DLR, 12489 Berlin, Germany. ${ }^{27}$ Università di Padova, 35131, Italy.

${ }^{28}$ INAF-IASF, 00133 Rome, Italy. ${ }^{29}$ IAS Orsay, 91405, France. ${ }^{30}$ LESIA, Observatoire de Paris, France. ${ }^{31}$ ASI Rome, 00198, Italy. ${ }^{32}$ Techno System Developments, Naples, 80078, Italy. ${ }^{33}$ Kayser Threde, Munich, 81379, Germany. 